\title{
Two Cases of Primary Ovarian Insufficiency Accompanied by High Serum Anti-Müllerian Hormone Levels and Preservation of Ovarian Follicles
}

\author{
Sungwook Chun ${ }^{1}$, Yun Hee $\mathrm{KoO}^{2}$, Yong Jin $\mathrm{Na}^{3}$ \\ ${ }^{1}$ Department of Obstetrics and Gynecology, Inje University Haeundae Paik Hospital, Busan, Korea, ${ }^{2}$ Busan Maria Fertility Clinic, Busan, \\ Korea, ${ }^{3}$ Department of Obstetrics and Gynecology, Pusan National University Yangsan Hospital, Yangsan, Korea
}

Primary ovarian insufficiency (POI) is defined as the presence of amenorrhea for $\geq 4$ months accompanied by evidence of two serum follicle-stimulating hormone levels in the menopausal range in women aged $<40$ years. Anti-Müllerian hormone (AMH) has been recognized as the most reliable marker of ovarian reserve status, and its serum level is very low or undetectable in women with POI. Here we report two cases of patients who were diagnosed with POI despite high serum AMH levels and preservation of ovarian follicles, as revealed by ultrasound. In addition, we have presented a review of the current literature regarding this condition.

Key Words: Anti-Müllerian hormone, Ovarian follicle, Primary ovarian insufficiency

\section{INTRODUCTION}

Primary ovarian insufficiency (POI) is defined as the presence of amenorrhea for $\geq 4$ months accompanied by two serum follicle-stimulating hormone (FSH) levels in the menopausal range for a woman $<$ than 40 years of age $[1,2]$. Approximately $1 \%$ of women will develop POI before the age of 40 years $[1,3]$. Women with untreated POI are at increased risk of developing osteoporosis, cardiovascular disease, dementia, and Parkinsonism [4]. Although premature follicular depletion is the cause in almost all cases, additional specific evaluation is indicated to exclude conditions (e.g., chromosomal and other genetic abnormalities and autoimmune diseases) that may have potential health implications for the patient [1]. If the diagnosis of POI is confirmed, the patient should be tested for FMR1 premutation, karyotype, and 21-hydroxylase antibody $[1,2]$.

Anti-Müllerian hormone (AMH) is now recognized as the most reliable marker of ovarian reserve status
$[1,5,6]$. Decreased serum AMH levels are a reflection of decreased ovarian reserve, and very low or undetectable AMH levels in women with amenorrhea strongly suggest ovarian failure [1,3,7]. In contrast, amenorrhea accompanied by AMH levels greater than $5 \mathrm{ng} / \mathrm{mL}$ is suggestive of polycystic ovary syndrome $[1,8]$.

We report two cases of patients diagnosed with POI at separate university hospitals who showed high serum $\mathrm{AMH}$ levels and the preservation of ovarian follicles on ultrasound.

\section{CASE REPORT}

\section{Case 1}

A 33-year-old woman was referred to a university hospital for the evaluation of secondary amenorrhea for 4 months. The patient had spontaneous thelarche at age 11 years and menarche at age 13 years. She denied experiencing vasomotor symptoms. A human chorionic gonadotropin (hCG) urine test was negative. The

Received: March 2, 2020 Revised: March 29, 2020 Accepted: May 3, 2020

Address for Correspondence: Yong Jin Na, Department of Obstetrics and Gynecology, Pusan National University Yangsan Hospital, 20 Geumoro, Mulgeum-eup, Yangsan 50612, Korea

Tel: 82-55-360-2130, E-mail: yjna@pusan.ac.kr, ORCID: https://orcid.org/0000-0001-6842-3139 
results of the physical examination were as follows: height $165.0 \mathrm{~cm}$, weight $60.2 \mathrm{~kg}$, systolic blood pressure $132 \mathrm{mmHg}$, and diastolic blood pressure $77 \mathrm{mmHg}$. Breast development and pubic hair growth were both stage 5 according to the Tanner staging system. Basal hormonal tests revealed highly elevated serum FSH and luteinizing hormone (LH) levels (52.7 IU/L and 33.2 IU/L, respectively), a low estradiol (E2) level (17.8 pg/ $\mathrm{mL})$, and high AMH level $(5.04 \mathrm{ng} / \mathrm{mL})$. Serum levels of prolactin, thyroid-stimulating hormone, and free $\mathrm{T} 4$ were all normal. A transvaginal ultrasound revealed a normal-sized uterus with polycystic ovarian morphology (> 20 antral follicles measuring 2-9 $\mathrm{mm}$ ) observed in the right ovary (Fig. 1A).

The patient was unmarried and wanted to preserve her fertility; therefore, she was advised to visit an infertility hospital for oocyte cryopreservation. The repeated basal hormonal tests were unchanged, and serum levels of androgens including total testosterone, free testosterone, $17 \alpha$-hydroxyprogesterone, and dehydroepiandrosterone sulfate were all normal. Induction of superovulation was attempted with a starting dose of 150 IU recombinant FSH (Follitrope ${ }^{\circledR}$; LG, Iksan, Korea) daily for oocyte cryopreservation. The dose of recombinant FSH was increased to 225 IU after one week because follicular development was not observed on ultrasound. However, follicular development was not observed despite the increase in FSH dose, and cycle cancellation was determined at that time. Further evaluation included karyotype, FMR1 premutation, and 21-hydroxylase antibody tests. The karyotype analysis revealed 47,XXX[1]/46,XX[29]; however, it was read as a normal variant by the specialist in laboratory medicine. The FMR1 premutation test revealed no abnormal CGG trinucleotide repeat expansion. The patient tested negative for 21-hydroxylase antibodies. In addition, anti-thyroglobulin antibodies and anti-thyroid peroxidase antibodies were negative. She was prescribed oral contraceptives $\left(\mathrm{Yaz}^{\circledR}\right)$ for hypoestrogenism associated with POI.

\section{Case 2}

A 25-year-old woman visited the primary gynecologic clinic because of amenorrhea for 6 months. She had frequently experienced hot flashes, night sweats, and insomnia. She experienced thelarche at age 12 years and menarche at age 14 years. Her menstruation had been irregular since menarche. A hCG urine test was negative. Basal endocrine testing revealed highly elevated serum FSH and LH levels (29.4 IU/L and 43.5 IU/L, respectively), a low E2 level $(25.5 \mathrm{pg} / \mathrm{mL})$, and normal prolactin level $(14.10 \mathrm{ng} / \mathrm{mL})$. Tests were repeated after 7 weeks and revealed serum FSH and E2 levels of 52.6 IU/L and $41.0 \mathrm{pg} / \mathrm{mL}$, respectively, and an AMH level of $10.88 \mathrm{ng} / \mathrm{mL}$. The patient was referred to a university hospital for further evaluation.

The initial physical examination at the university hospital revealed the following: height $156.8 \mathrm{~cm}$, weight $66.9 \mathrm{~kg}$, systolic blood pressure $120 \mathrm{mmHg}$, diastolic blood pressure $80 \mathrm{mmHg}$, pulse rate $20 / \mathrm{min}$, respiratory rate $80 / \mathrm{min}$, and body temperature $36.4^{\circ} \mathrm{C}$. Breast development and pubic hair growth were both stage 5 according to the Tanner classification system. A transrectal ultrasound revealed a normal-sized uterus with polycystic ovarian morphology (35 antral follicles measuring 2-9 $\mathrm{mm}$ in the right ovary [Fig. 1B] and 26 in the left ovary). The repeated serum FSH, LH, E2, and $\mathrm{AMH}$ levels acquired in the university hospital were unchanged. Elevated thyroid stimulating hormone (TSH; $6.63 \mathrm{mIU} / \mathrm{L})$ and normal free T4 (1.07 ng/dL) levels were observed, and all anti-thyroid antibody tests showed above normal blood levels of antibodies (anti-
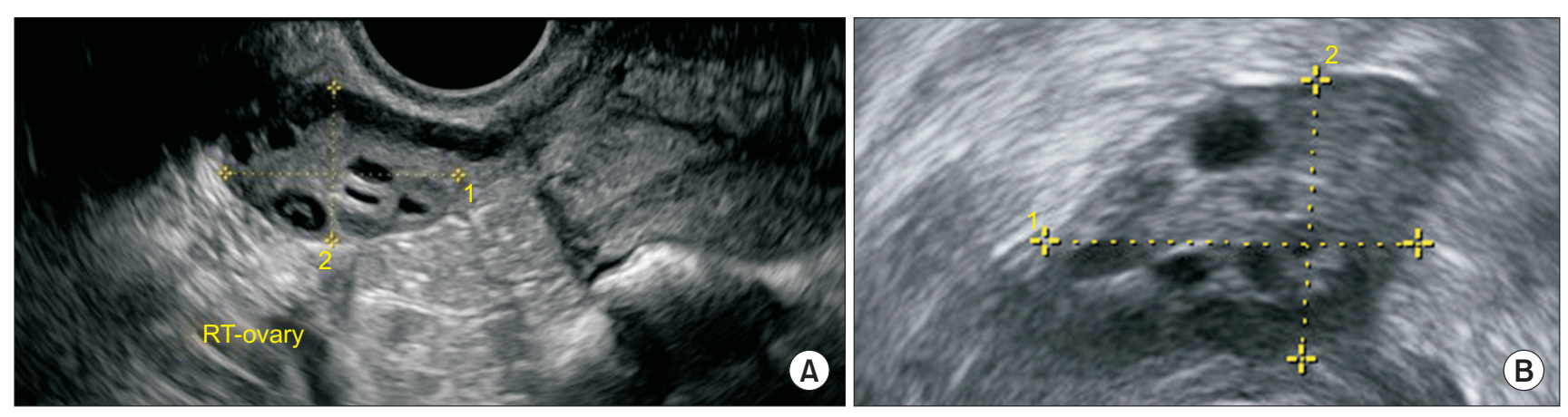

Fig. 1. Ultrasound findings of right (RT) ovary in: (A) Case 1; (B) Case 2. 
thyroglobulin antibody $234.6 \mathrm{IU} / \mathrm{mL}$ and anti-thyroid peroxidase antibody $845.1 \mathrm{IU} / \mathrm{mL}$ ). Serum androgen levels were normal. A karyotype analysis revealed a normal female karyotype. The FMR1 premutation test was normal, and the 21-hydroxylase antibody test was negative. She was prescribed conjugated estrogens $\left(\right.$ Premina $\left.^{\circledR}\right) 0.625 \mathrm{mg} / \mathrm{d}$ in combination with cyclic micronized progestogen (Utrogestan ${ }^{\circledR}$ ) $200 \mathrm{mg}$ daily for 14 days per month.

\section{DISCUSSION}

Women with amenorrhea younger than 40 years of age with an evidence of persisting hypergonadotropic hypogonadism are diagnosed with POI [1]. Diagnosis should be confirmed by an elevated FSH level greater than $40 \mathrm{IU} / \mathrm{L}$ and an E2 level below $50 \mathrm{pmol} / \mathrm{L}$ in the absence of bilateral oophorectomy [4].

Circulating levels of $\mathrm{AMH}$ are now recognized as a more reliable marker of ovarian reserve status compared to $\mathrm{FSH}$, inhibin $\mathrm{B}$, and the antral follicle count $[1,5,6]$. AMH levels have been alleged to be very low or undetectable in women with POI $[3,5,7]$. However, in the present two cases, both POI patients had high serum $\mathrm{AMH}$ levels (> $5 \mathrm{ng} / \mathrm{mL}$ ).

One possible explanation is that the cause of POI in the present case reports is steroidogenic autoimmunity. Approximately $4 \%$ of women with POI were noted to have steroidogenic cell autoimmunity (SCA) with lymphocytic oophoritis (autoimmune oophoritis) as the mechanism for follicular dysfunction $[1,2,9]$. Some studies noted that women with POI due to SCA (SCAPOI) had normal serum AMH levels $[3,7,10,11]$. La Marca et al. [11] reported that AMH concentrations were significantly higher in women with SCA-POI than in women with idiopathic POI or postmenopausal women, and two thirds of women with recent-onset ( $<5$ years) SCA-POI had normal serum AMH levels. Normal serum AMH levels in SCA-POI suggest that the pool of growing follicles in autoimmune POI is initially preserved $[7,12]$. Ovarian antibody testing is not clinically reliable for diagnosing autoimmune oophoritis because some women with biopsy-proven autoimmune oophoritis may have a negative test for ovarian antibodies [2]. However, women with autoimmune lymphocytic oophoritis appear to reliably test positive for adrenal antibodies, and the most readily available antibody is 21-hydroxylase. In our cases, both patients tested negative for the 21-hydroxylase antibody; thus, autoimmune oophoritis is hardly considered as a strong causative factor.

Several other autoimmune disorders have also been associated with POI; hypothyroidism is the most common disorder [13], but there are no direct evidences of relationship between hypothyroidism and its effect against POI [1]. In addition to testing for the 21-hydroxylase antibody, women who are suspected of having POI should be screened for anti-thyroid antibodies (antithyroid peroxidase and antithyroglobulin antibodies) $[1,2]$. In case 2 , all anti-thyroid antibodies were positive along with the elevation of serum TSH levels; however, the presence of thyroid autoantibodies does not prove autoimmune ovarian failure [2].

Another possible (and seemingly more attractive) explanation concerning our cases is that a FSH receptor mutation or FSH-resistant ovaries (FSHRO) induced POI in our patients. Disproportionately elevated FSH levels can be observed despite the presence of ovarian follicles in cases of inactivating mutations involving the FSH or LH receptors [1]. Kallio et al. [10] previously identified a recessively inherited inactivating mutation in the FSH receptor gene, which results in a dramatic reduction in signal transduction and FSHRO. Additionally, they reported that serum levels of AMH in women with FSHRO were not significantly different from those in normal control women and significantly higher than levels in women with primary or secondary amenorrhea of unknown etiology [10].

Gonadotropin-resistant ovary syndrome (gonadotropin-ROS) or ROS, first described by Jones and De Moraes-Ruehsen [14] in 1969, is known to be one of the causes of ovarian failure leading to primary or secondary amenorrhea. The presence of a normal amount of antral follicles is a criterion that distinguishes ROS from the classical POI $[14,15]$. Besides an age-compatible number of small antral follicles, additional cardinal clinical features of ROS are normal chromosome, elevated gonadotropin levels of menopausal range, and unresponsiveness to gonadotropin stimulation [15], all of which are consistent with our cases. If the diagnosis of our cases is correct for ROS, in vitro maturation (IVM) of oocytes may be a feasible option of treatment for infertility in our cases because some authors reported successful live births in women with ROS following IVM using their own oocytes $[15,16]$.

In case 2, the chromosomal karyotyping test revealed $47, \mathrm{XXX}[1] / 46, \mathrm{XX}[29]$; however, this result was read as a normal variant because $1 \%-2 \%$ of $47, \mathrm{XXX}$ cells 
could be found in normal women [17]. Women with a 47,XXX karyotype may develop ovarian failure [2], and even low-level X-mosaicism (between $6 \%-10 \%$ of aneuploid cells) might be associated with the development of POI [17].

$\mathrm{AMH}$ is an undoubtedly useful marker for the assessment of ovarian reserve, and it also may be a feasible marker for the diagnosis of POI. However, in rare situations, normal or high serum AMH levels may occur in patients who are diagnosed with POI. Further studies are needed to clarify the utility of serum AMH levels in women suspected of having POI.

\section{CONFLICT OF INTEREST}

No potential conflict of interest relevant to this article was reported.

\section{REFERENCES}

1. Taylor HS, Pal H, Seli E. Speroff's clinical gynecologic endocrinology and infertility. 8th ed. Philadelphia (PA): Lippincott Williams \& Wilkins; 2019.pp.343-94.

2. Baker VL, Beall SA. Amenorrhea. In: Berek JS, editor. Berek \& Novak's gynecology. 16th ed. Philadelphia (PA): Lippincott Williams \& Wilkins; 2019. pp.866-88.

3. La Marca A, Pati M, Orvieto R, Stabile G, Carducci Artenisio A, Volpe A. Serum anti-müllerian hormone levels in women with secondary amenorrhea. Fertil Steril 2006; 85: 1547-9.

4. Vujovic S, Brincat M, Erel T, Gambacciani M, Lambrinoudaki I, Moen $\mathrm{MH}$, et al. EMAS position statement: managing women with premature ovarian failure. Maturitas 2010; 67: 91-3.

5. Knauff EA, Eijkemans MJ, Lambalk CB, ten Kate-Booij MJ, Hoek A, Beerendonk CC, et al. Anti-Mullerian hormone, inhibin B, and antral follicle count in young women with ovarian failure. J Clin Endocrinol Metab 2009; 94: 786-92.

6. Lee JR, Kim SH. Anti-Mullerian hormone and female reproduction. Korean J Obstet Gynecol 2009; 52: 285-300.

7. Visser JA, Schipper I, Laven JS, Themmen AP. Anti-Müllerian hormone: an ovarian reserve marker in primary ovarian insuffi- ciency. Nat Rev Endocrinol 2012; 8: 331-41.

8. Dewailly D, Gronier H, Poncelet E, Robin G, Leroy M, Pigny P, et al. Diagnosis of polycystic ovary syndrome (PCOS): revisiting the threshold values of follicle count on ultrasound and of the serum $\mathrm{AMH}$ level for the definition of polycystic ovaries. Hum Reprod 2011; 26: 3123-9.

9. Bakalov VK, Anasti JN, Calis KA, Vanderhoof VH, Premkumar A, Chen $\mathrm{S}$, et al. Autoimmune oophoritis as a mechanism of follicular dysfunction in women with 46,XX spontaneous premature ovarian failure. Fertil Steril 2005; 84: 958-65.

10. Kallio S, Aittomäki K, Piltonen T, Veijola R, Liakka A, Vaskivuo TE, et al. Anti-Mullerian hormone as a predictor of follicular reserve in ovarian insufficiency: special emphasis on FSH-resistant ovaries. Hum Reprod 2012; 27: 854-60.

11. La Marca A, Marzotti S, Brozzetti A, Stabile G, Artenisio AC, Bini V, et al. Primary ovarian insufficiency due to steroidogenic cell autoimmunity is associated with a preserved pool of functioning follicles. J Clin Endocrinol Metab 2009; 94: 3816-23.

12. Massin N, Méduri G, Bachelot A, Misrahi M, Kuttenn F, Touraine P. Evaluation of different markers of the ovarian reserve in patients presenting with premature ovarian failure. Mol Cell Endocrinol 2008; 282: 95-100.

13. Komorowska B. Autoimmune premature ovarian failure. Prz Menopauzalny 2016; 15: 210-4.

14. Jones GS, De Moraes-Ruehsen M. A new syndrome of amenorrhae in association with hypergonadotropism and apparently normal ovarian follicular apparatus. Am J Obstet Gynecol 1969; 104 : 597-600.

15. Li Y, Pan P, Yuan P, Qiu Q, Yang D. Successful live birth in a woman with resistant ovary syndrome following in vitro maturation of oocytes. J Ovarian Res 2016; 9: 54.

16. Grynberg M, Peltoketo H, Christin-Maitre S, Poulain M, Bouchard P, Fanchin R. First birth achieved after in vitro maturation of oocytes from a woman endowed with multiple antral follicles unresponsive to follicle-stimulating hormone. J Clin Endocrinol Metab 2013; 98: 4493-8.

17. Gersak K, Veble A. Low-level X chromosome mosaicism in women with sporadic premature ovarian failure. Reprod Biomed Online 2011; 22: 399-403. 